\title{
A Randomized, Double-Blind, Placebo-Controlled Phase III Trial On The Efficacy and Safety of Tocilizumab in Patients With Colchicine-Resistant or -Intolerant Familial Mediterranean Fever
}

\section{Tomohiro Koga ( $\nabla$ tkoga@nagasaki-u.ac.jp )}

Nagasaki University https://orcid.org/0000-0003-2077-4428

Shuntaro Sato

Nagasaki University: Nagasaki Daigaku

Naoko Hagimori

Nagasaki University: Nagasaki Daigaku

Hiroshi Yamamoto

Nagasaki University: Nagasaki Daigaku

Masataka Ishimura

Kyushu University: Kyushu Daigaku

Takahiro Yasumi

Kyoto University: Kyoto Daigaku

Yohei Kirino

Yokohama City University: Yokohama Shiritsu Daigaku

Kei Ikeda

Chiba University: Chiba Daigaku

Akihiro Yachie

Kanazawa University: Kanazawa Daigaku

Kiyoshi Migita

Fukushima Kenritsu Ika Daigaku

Dai Kishida

Shinshu Daigaku

Tatsuya Atsumi

Hokkaido University

Atsushi Kawakami

Nagasaki University: Nagasaki Daigaku

Research article 
Keywords: IL-6, placebo, tocilizumab, FMF, colchicine-resistant

Posted Date: October 27th, 2021

DOI: https://doi.org/10.21203/rs.3.rs-1005662/v1

License: (c) (1) This work is licensed under a Creative Commons Attribution 4.0 International License. Read Full License 


\section{Abstract \\ Objective}

To evaluate the efficacy and safety of tocilizumab (TCZ), an interleukin 6 receptor monoclonal antibody, in patients with familial Mediterranean fever (FMF).

\section{Methods}

We performed a double-blind, randomized, parallel-group trial, followed by an open-label extension trial, in patients with colchicine-resistant or -intolerant FMF (crFMF). Patients were randomly assigned (1:1) to receive TCZ (162 mg every week) or placebo, administered subcutaneously, for 24 weeks. Rescue treatment was allowed if the rescue criteria were met. The primary endpoint was the number of fever attacks over the 24 weeks of treatment. Secondary endpoints included the frequency of accompanying symptoms during attacks, serum CRP and SAA values, and adverse events (AEs). The open-label extension study evaluated the long-term safety and efficacy of TCZ in patients who had completed the preceding study.

\section{Results}

We randomly assigned 23 patients to either TCZ $(n=11)$ or placebo $(n=12)$. The TCZ-placebo rate ratios were 0.691 (95\% confidence intervals $(\mathrm{Cl}), 0.189-2.531 ; P=0.577)$ for the fever attacks, based on the group rates per week. The recurrence of attacks was significantly lower in the TCZ group (hazard ratio $=0.457 ; 95 \% \mathrm{Cl}, 0.240-0.869)$. Fever attacks, accompanying symptoms, serum CRP and SAA values were controlled in most of the patients who received long-term TCZ. In these trials, the numbers and severity of AEs did not differ between groups.

\section{Conclusion}

Although a primary endpoint was not met in the preceding trial, long-term administration of TCZ showed stable efficacy and safety for patients with crFMF.

\section{Introduction}

Familial Mediterranean fever (FMF) is the most common hereditary autoinflammatory disorder, and is characterized by recurrent attacks of fever with arthritis, skin rash, and serositis [1, 2]. FMF is caused by a number of mutations of the Mediterranean Fever (MEFV) gene, coding a 781-amino acid protein called pyrin that acts as a major regulatory component of the inflammasome [3]. Accordingly, the pathological condition of FMF is thought to be mainly due to mutations of pyrin that cause abnormal activation of the inflammasome $[4,5]$. 
The therapeutic goal of FMF is to prevent secondary amyloidosis by minimizing subclinical inflammation between attacks, in addition to preventing acute attacks, and colchicine has been recommended as a first-line treatment for adults and children $[6,7]$. However, $10-20 \%$ of FMF patients do not respond well to colchicine or discontinue use due to adverse effects $[8,9]$.

Although activation of the inflammasome pathway by the $M E F V$ variant-induced dysfunctions of pyrin is the main pathological mechanism of FMF [4,5], FMF patients have elevated serum levels of inflammatory cytokines such as interleukin (IL)-1ß, IL-6, IL-17, and IL-18 [10-13]. These cytokines activate nuclear factor $\mathrm{KB}$ signaling pathways, which subsequently lead to increased amounts of pro-IL-1 $\beta$, tumor necrosis factor-alpha (TNF-a), and IL-6 $[10,11]$. Thus, using biologic agents to block these cytokines is considered to be a reasonable approach for the management of FMF [14-16].

Several reports have shown the efficacy of an IL-6 inhibitor in clinical practice for colchicine-resistant FMF or secondary amyloidosis in FMF patients [17-23]. However, there have been no randomized, placebo-controlled trials of anti-IL-6 treatment in patients with FMF. This phase III study was conducted to confirm the beneficial effects of tocilizumab (TCZ) in patients with colchicine-resistant or -intolerant FMF (crFMF).

\section{Methods}

\section{Study design}

This investigator-initiated, multicenter, double-blind, randomized, placebo-controlled phase III trial was conducted at 9 centers in Japan. This study is registered on the University Hospital Medical Information Network Clinical Trials Registry as UMIN000028010. The protocol of this trial (NUH01FMF) was published previously [24]. There were no substantial changes to the published study design, methods, or outcomes after the start of the trial.

Patients were recruited from 1 March 2018 to 31 December 2019 and were randomly assigned 1:1 to receive weekly TCZ (162 mg) or placebo subcutaneously. During the double-blind period, patients with more than 4 fever attacks had TCZ introduced by subcutaneous injection for the rescue treatment.

All patients who completed the double-blind phase (NUH01FMF) were transferred to the TCZ arm and enrolled in the extension study (NUH02FMF). The detailed protocol for the extension study has already been reported [25].

\section{Patients}

Eligible patients were 12 to 75 years old; had been diagnosed with typical FMF based on the Tel Hashomer criteria [26, 27], and were resistant to or intolerant of colchicine treatment. The frequency of attacks was collected from the time of obtaining patient consent until 24 weeks prior and by referring to the electronic medical record. Colchicine resistance was defined as the occurrence of at least one fever attack in 3 months even after increasing the dose to the maximum level (1.5-2.0 mg/day). Colchicine 
intolerance was defined as inability to continue the drug or to increase the maximum dose (1.5-2.0 $\mathrm{mg} /$ day) due to side effects such as gastrointestinal symptoms and fever attacks occurring more than once in 3 months. The definitions of colchicine resistance and intolerance used in this study were consistent with the consensus recently outlined by Ozen et al. [28]. After the provisional registration, patients who had a fever attack due to FMF were randomly assigned to the TCZ group or the placebo group.

Patients were excluded if they changed doses of an oral corticosteroid or colchicine during the doubleblind period, or if they took a prednisolone dose $>5 \mathrm{mg} /$ day. Patients were also excluded if they received corticosteroids intravenously or intramuscularly, biologics, non-steroidal anti-inflammatory drugs, or acetaminophen during the double-blind period. Other inclusion and exclusion criteria are previously described [24, 25]. All patients provided written informed consent prior to their enrollment in the study. The study protocol was approved by the Institutional Review Board of Nagasaki University and other participating centers.

\section{Assessments and outcomes}

The primary endpoint was the number of fever attacks due to FMF over 24 weeks of treatment. For this clinical trial, a fever attack was defined as having a fever $>38.0^{\circ} \mathrm{C}$ lasting $\geq 6$ hours. The secondary endpoints were the frequency of accompanying symptoms during attacks; the time between fever attacks; the durations of fever attacks; the serum CRP and SAA values; the patient's score on the 36-item short form health survey (SF-36) questionnaire; results of a general evaluation by a physician $(100 \mathrm{~mm}$ Visual Analog Scale [VAS]); body temperature; and the percentage of achievement of FMF 50 score (improvement of $50 \%$ or more in at least five of six items) [29] at 12 weeks and 24 weeks during the double-blind phase. Patients were monitored for safety including the adverse events and a pharmacodynamic assessment throughout the NUH01FMF and NUH02FMF trial.

\section{Statistical analyses}

Details of the statistical analysis were described previously, and we changed only the estimator of the interval estimates obtained from the negative binomial regression analysis before the key open, which is also used for the primary analysis [24, 25]. We estimated that a sample size of 24 patients (12 per group) would provide $\geq 80 \%$ power for the between-group comparison of the primary endpoint (i.e., the number of fever attacks over 24 weeks of treatment), assuming averages of 1.5 fever attacks in the TCZ group and 6 in the placebo group and a 2-sided alpha level of 0.05, based on the negative binomial distribution. Negative binomial dispersion parameters of the TCZ and placebo groups were assumed to be 4 and 2.6, respectively.

Baseline patient characteristics were summarized within the TCZ and placebo groups. Continuous variables are shown as means with standard deviations (SDs) or medians with interquartile ranges (IQRs). Categorical variables are shown as the number of patients and percentages within each group. 
For the efficacy analysis, we used the full population from the double-blind period. Using a negative binomial regression analysis with the number of fever attacks (the primary endpoint) as the outcome variable, the drug (TCZ or placebo) as the explanatory variable, and the double-blind period as the offset term, we estimated the fever attack rates (the number of fever attacks per week) in the TCZ and placebo groups and the rate ratio of TCZ to placebo. We tested and calculated $95 \%$ confidence intervals (Cls) using a sandwich variance to ensure validity for model misspecification. Secondary endpoints were analyzed in the same way as the primary endpoint. Additionally, we considered the number of fever attacks over the 24 weeks of treatment as a recurrent event, and performed the counting process approach using the stratified Cox proportional hazard model. Statistical tests were 2-sided, and $P$ values $<0.05$ were considered to be significant for the primary endpoint. Unique laboratory values in this study are summarized and illustrated. The post-rescue data were considered missing and imputed by the last observation carried forward method.

We used SAS version 9.2 (SAS Institute, Cary, North Carolina) to create the data set and perform statistical analyses, and created figures using R version 4.0.2.

\section{Results}

\section{Patient flow and characteristics}

Of the 49 screened patients, 23 were enrolled in the study. We randomly assigned 12 patients to the placebo group and 11 to the TCZ group (Figure 1). Of the patients who were excluded before enrollment due to a 12-week absence of fever attacks during the observation period, 2 patients who regained consent were found to have a fever attack during the observation period and met the enrollment criteria. Both of these patients were randomly assigned to the placebo group. Baseline demographic and disease characteristics were balanced between the groups (Table 1), but patients who received TCZ were slightly younger and had higher levels of CRP, SAA, and IL- 6 at baseline. There were 4 patients from the placebo group and 1 from the TCZ group who met the criteria for rescue treatment. 
Table 1

Baseline patient characteristics

\begin{tabular}{|c|c|c|}
\hline & Placebo $(n=12)$ & Tocilizumab $(n=11)$ \\
\hline Age at enrollment, years & $45.9(11.0)$ & $37.5(14.5)$ \\
\hline Age at diagnosis, years & $41.9(13.1)$ & $33.3(16.0)$ \\
\hline Female, n (\%) & $6(50)$ & $8(73)$ \\
\hline Height, cm & $160.5(7.4)$ & $161.2(7.5)$ \\
\hline Body weight, $\mathrm{kg}$ & $60.9(12.1)$ & $54.1(11.8)$ \\
\hline 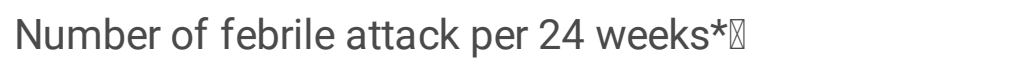 & $4.88(2.57-8.87)$ & $5.14(2.75-8.31)$ \\
\hline Duration of febrile attack, hours* & $44.4(32.9-103)$ & $55.3(38.6-83.6)$ \\
\hline Patient global VAS, mm & $23.5(26.3)$ & $37.6(23.7)$ \\
\hline Physician global VAS, mm & $35.0(28.8)$ & $39.2(29.7)$ \\
\hline CRP, mg/L & $7.06(13.5)$ & $11.8(24.5)$ \\
\hline $\mathrm{SAA}, \mathrm{mg} / \mathrm{L}$ & $468(1000)$ & $1140(3270)$ \\
\hline IL-6, ng/L & $3.63(4.88)$ & $12.92(29.3)$ \\
\hline Number of febrile attacks with arthritis per 24 weeks $\star \nabla$ & $1.45(0.36-4.95)$ & $2.75(1.60-8.31)$ \\
\hline Dose of colchicine, mg/day & $0.63(0.68)$ & $0.59(0.54)$ \\
\hline \multicolumn{3}{|l|}{ MEFV gene variants ${ }^{\ddagger}$} \\
\hline Exon 10 mutations, total, $\mathrm{n}(\%)$ & $3(30)$ & $0(0)$ \\
\hline M694I hetero, n (\%) & $2(20)$ & $0(0)$ \\
\hline M694I/E148Q multiple hetero, n (\%) & $1(10)$ & $0(0)$ \\
\hline Any other mutations, total, n (\%) & $6(60)$ & $6(86)$ \\
\hline E84K hetero, n (\%) & $2(20)$ & $0(0)$ \\
\hline
\end{tabular}

Data are $\mathrm{n} / \mathrm{N}(\%)$ or mean (SD) unless otherwise noted.

*Median (IQR).

QFrom 24 weeks before informed consent to the first dosing period.

₹Placebo $n=10 ;$ Tocilizumab $n=7$.

CRP, C-reactive protein; IL, interleukin; IQR, interquartile range; SAA, serum amyloid A; SD, standard deviation; VAS, Visual Analogue Scale 


\begin{tabular}{|c|c|c|}
\hline & Placebo $(n=12)$ & Tocilizumab $(n=11)$ \\
\hline E84K/E148Q multiple hetero, $\mathrm{n}(\%)$ & $1(10)$ & $0(0)$ \\
\hline E148Q hetero, n (\%) & $5(50)$ & $4(57)$ \\
\hline P369S/R408Q multiple hetero, $\mathrm{n}(\%)$ & $0(0)$ & $2(39)$ \\
\hline G304R hetero, $n(\%)$ & $1(10)$ & $0(0)$ \\
\hline \multicolumn{3}{|c|}{ Data are $\mathrm{n} / \mathrm{N}(\%)$ or mean (SD) unless otherwise noted. } \\
\hline \multicolumn{3}{|l|}{ *Median (IQR). } \\
\hline \multicolumn{3}{|c|}{ هFrom 24 weeks before informed consent to the first dosing period. } \\
\hline \multicolumn{3}{|l|}{${ }^{\ddagger}$ Placebo $n=10 ;$ Tocilizumab $n=7$} \\
\hline \multicolumn{3}{|c|}{$\begin{array}{l}\text { CRP, C-reactive protein; IL, interleukin; IQR, interquartile range; SAA, serum amyloid A; SD, standard } \\
\text { deviation; VAS, Visual Analogue Scale }\end{array}$} \\
\hline
\end{tabular}

\section{Primary outcome in 24-week, double-blind phase (NUH01FMF trial)}

Figure 2 shows the number of fever attacks during the double-blind period of the study. Occurred over 4 weeks, there were an estimated 0.37 attacks in the TCZ group versus 0.53 in the placebo group. Negative binomial regression analyses showed that the estimated number of fever attacks per 1 week was 0.078 (95\% Cl, 0.027-0.222) in the TCZ group and $0.113(95 \% \mathrm{Cl}, 0.053-0.242)$ in the placebo group, and the TCZ-placebo attack rate ratio was $0.691(95 \% \mathrm{Cl}, 0.189-2.531)$. Although the number of attacks was lower in the TCZ group than in the placebo group, the results were not significant $(P=0.58)$.

\section{Secondary outcomes in 24-week, double-blind phase (NUH01FMF trial)}

Table 2 shows occurrences of symptoms accompanying attacks, the time between fever attacks, and the durations of fever attacks. Negative binomial regression analyses revealed that the estimated number of any accompanying symptom during attacks per 1 week was 0.147 in the TCZ group and 0.274 in the placebo group (the TCZ-placebo rate ratio $=0.538 ; 95 \% \mathrm{Cl}, 0.176-1.644$ ). Abdominal pain, chest pain, and headache tended to be less common in the TCZ group, but there were no significant differences between the two groups regarding arthritis, the time between fever attacks, or the duration of fever attacks (Table 2). The percentage of patients who entered the rescue phase was $33.3 \%$ in the placebo group compared to $9.1 \%$ in the TCZ group, according to the Kaplan-Meier estimate. In the recurrence event data analysis for fever attack using the stratified counting process approach, there were significantly fewer attacks during the double-blind period in the TCZ group (hazard ratio $=0.457 ; 95 \% \mathrm{Cl}$, 0.240-0.869). 
Table 2

Secondary outcomes in 24-week, double-blind phase (NUH01FMF trial)

\begin{tabular}{|c|c|c|c|}
\hline & Placebo $(n=12)$ & $\begin{array}{l}\text { Tocilizumab }(n= \\
\text { 11) }\end{array}$ & $\begin{array}{l}\text { TCZ-placebo rate } \\
\text { ratio }\end{array}$ \\
\hline \multicolumn{4}{|c|}{ Accompanying symptoms, times/week } \\
\hline Any & $\begin{array}{l}0.274(0.128- \\
0.586)\end{array}$ & $\begin{array}{l}0.147(0.065- \\
0.334)\end{array}$ & $0.538(0.176-1.644)$ \\
\hline Abdominal pain & $\begin{array}{l}0.058(0.024- \\
0.140)\end{array}$ & $\begin{array}{l}0.033(0.014- \\
0.075)\end{array}$ & $0.570(0.170-1.914)$ \\
\hline Chest pain & $\begin{array}{l}0.076(0.024- \\
0.242)\end{array}$ & $\begin{array}{l}0.016(0.004- \\
0.067)\end{array}$ & $0.215(0.034-1.338)$ \\
\hline Arthritis & $\begin{array}{l}0.058(0.024- \\
0.142)\end{array}$ & $\begin{array}{l}0.055(0.012- \\
0.243)\end{array}$ & $0.944(0.167-5.344)$ \\
\hline Headache & $\begin{array}{l}0.054(0.021- \\
0.137)\end{array}$ & $\begin{array}{l}0.025(0.009- \\
0.070)\end{array}$ & $0.456(0.113-1.842)$ \\
\hline $\begin{array}{l}\text { Time until a fever attack, } \\
\text { hours* }\end{array}$ & $794(580-914)$ & $907(323-1019)$ & - \\
\hline $\begin{array}{l}\text { Duration of fever attacks, } \\
\text { hours* }\end{array}$ & $45(34-100)$ & $72(66-110)$ & - \\
\hline \multicolumn{4}{|l|}{$95 \% \mathrm{Cl}$ of Estimate. } \\
\hline \multicolumn{4}{|l|}{ *Median (IQR). } \\
\hline $\mathrm{Cl}$, confidence interval; IQR & artile range; $\mathrm{TC}$ & zumab. & \\
\hline
\end{tabular}

The median changes from baseline in serum CRP and SAA values are shown in Figure $3 \mathrm{~A}$ and $\mathrm{B}$, respectively. In the TCZ group, the median serum CRP level was $0.70 \mathrm{mg} / \mathrm{L}$ (range, $0.20-82 \mathrm{mg} / \mathrm{L}$ ) at baseline, decreased to $0.20 \mathrm{mg} / \mathrm{L}$ (range, $0.2-6.3 \mathrm{mg} / \mathrm{L}$ ) at 2 weeks after treatment start, and became negative $(<1.0 \mathrm{mg} / \mathrm{L})$ in all patients $(0.20 \mathrm{mg} / \mathrm{L}$; range, $0.20-0.80 \mathrm{mg} / \mathrm{L})$ at 8 weeks. In the placebo group, CRP level was $2.15 \mathrm{mg} / \mathrm{L}$ (range, $0.20-46 \mathrm{mg} / \mathrm{L}$ ) at baseline and was $1.65 \mathrm{mg} / \mathrm{L}(0.20-3.8 \mathrm{mg} / \mathrm{L}$ ) at 24 weeks, which was abnormal in some patients as those in the baseline. Similarly, the median serum SAA level in the TCZ group was $7.5 \mathrm{mg} / \mathrm{L}$ (range, $2.5-1100 \mathrm{mg} / \mathrm{L}$ ) at baseline, but decreased to $2.7 \mathrm{mg} / \mathrm{L}$ (range, 2.5-41 $\mathrm{mg} / \mathrm{L}$ ) at 2 weeks and remained lower than baseline thereafter. In the placebo group, the baseline level was $6.6 \mathrm{mg} / \mathrm{L}$ (range, 2.5-350 mg/L), but it was $8.8 \mathrm{mg} / \mathrm{L}$ (range, $2.5-170 \mathrm{mg} / \mathrm{L}$ ) at 2 weeks and $6.3 \mathrm{mg} / \mathrm{L}$ (range, $2.5-55 \mathrm{mg} / \mathrm{L}$ ) at 24 weeks, remaining near the baseline even after treatment.

At 24 weeks, $2 / 10$ patients (20.0\%) in the TCZ group and 1/12 (8.3\%) in the placebo group achieved FMF50 [29]. The main changes in the FMF50 core set during the double-blind phase were improvement from baseline in all items in the TCZ group at 24 weeks and a decrease in scores compared to the 
placebo group. The amount of change and percentage change for each item in the FMF core set are shown in Supplementary Table 1. The mean change from baseline in the physician VAS was not significantly different between two groups (Figure 3C).

The statistics for summary measures of the SF-36 analysis are shown in Supplementary Figure 1. At 24 weeks, the TCZ and placebo groups improved by 10 points or more in daily functioning (physical; 12.5 and 18.8, respectively) and in body pain (42.0 in the placebo group), but there was no difference between the two groups in other items, including the summary scale.

\section{Efficacy in 48-week, open-label phase (NUH02FMF trial)}

In the NUH01FMF trial, the TCZ arm had a mean number of fever attacks per week ( \pm SD) during the double-blind period of $0.093 \pm 0.192$. The mean numbers of fever attacks per week were $0.176 \pm 0.266$, $0.0898 \pm 0.149,0.079 \pm 0.148$, and $0.068 \pm 0.153$ at 4, 12, 24, and 48 weeks, respectively, after the start of TCZ treatment. These results suggest that most patients tend to have fewer fever attacks in the long term. In the NUH01FMF trial, the placebo arm had a mean number of fever attacks per week during the double-blind period of $0.132 \pm 0.204$, and the mean numbers of fever attacks per week after the start of TCZ were $0.00 \pm 0.00,0.04 \pm 0.119,0.049 \pm 0.130$, and $0.041 \pm 0.088$ at $4,12,24$, and 48 weeks, respectively, suggesting a long-term trend toward fewer fever attacks in most patients. The number of fever attacks tended to decrease as the duration of TCZ treatment increased.

Similarly, there was a tendency for accompanying fever attack symptoms to be suppressed over time. Supplementary Table 2 shows the trend of accompanying symptoms in the extension study. In addition, serum CRP levels became negative over the long term, and serum SAA levels remained significantly lower than the baseline levels after the start of TCZ treatment. The trends for serum CRP, SAA, and physician VAS data after TCZ administration in all patients during the double-blind period and the extension study are shown in Supplementary Figure 2.

In summary, fever attacks were controlled in most of the patients who received long-term TCZ and those who switched to TCZ, suggesting the efficacy of TCZ in patients with FMF.

\section{Safety (NUH01FMF and NUH02FMF trial)}

Table 3 shows summaries of the adverse events during the double-blind period, in patients treated with TCZ during the rescue period, and during the entire period. The total number of adverse events during the double-blind period is shown in Supplementary Table 3. The major adverse events were hypofibrinogenemia (8 cases), injection site reaction ( 2 cases), and headache (2 cases) in the TCZ group, and folliculitis and upper respiratory tract inflammation ( 2 cases each) in the placebo group. In the placebo group, there were 2 cases each of folliculitis and upper respiratory tract inflammation. There were no adverse events that led to death throughout the study period. 
Table 3

The summary of adverse events

\begin{tabular}{|c|c|c|}
\hline & \multirow{2}{*}{$\begin{array}{l}\text { Tocilizumab } \\
\text { Case (\%) }\end{array}$} & \multirow{2}{*}{$\begin{array}{l}\text { Placebo } \\
\text { Case (\%) }\end{array}$} \\
\hline & & \\
\hline Double blind period & $\mathrm{n}=11$ & $\mathrm{n}=12$ \\
\hline Adverse event & $11(100)$ & $8(66.7)$ \\
\hline Adverse event leading to death & $0(0)$ & $0(0)$ \\
\hline Adverse event leading to withdrawal from treatment & $1(9.1)$ & $0(0)$ \\
\hline Serious adverse event & $2(18.2)$ & $1(8.3)$ \\
\hline Serious adverse event leading to withdrawal from treatment & $1(9.1)$ & $0(0.0)$ \\
\hline Adverse drug reactions & $10(90.9)$ & $4(33.3)$ \\
\hline Serious adverse drug reactions & $1(9.1)$ & $0(0)$ \\
\hline Adverse drug reactions leading to withdrawal from treatment & $1(9.1)$ & $0(0)$ \\
\hline Rescue period & $\mathrm{n}=1$ & $\mathrm{n}=4$ \\
\hline Adverse event & $1(100.0)$ & $3(75.0)$ \\
\hline Adverse event leading to death & $0(0)$ & $0(0)$ \\
\hline Adverse event leading to withdrawal from treatment & $0(0)$ & $0(0)$ \\
\hline Serious adverse event & $0(0)$ & $2(50)$ \\
\hline Serious adverse event leading to withdrawal from treatment & $0(0)$ & $0(0)$ \\
\hline Adverse drug reactions & $1(100)$ & $3(75)$ \\
\hline Serious adverse drug reactions & $0(0)$ & $2(50)$ \\
\hline Adverse drug reactions leading to withdrawal from treatment & $0(0)$ & $0(0)$ \\
\hline Tocilizumab treatment period & $\mathrm{n}=11$ & $\mathrm{n}=4$ \\
\hline Adverse event & $11(100)$ & $3(75)$ \\
\hline Adverse event leading to death & $0(0)$ & $0(0)$ \\
\hline Adverse event leading to withdrawal from treatment & $1(9.1)$ & $0(0)$ \\
\hline Serious adverse event & $2(18.2)$ & $2(50)$ \\
\hline Serious adverse event leading to withdrawal from treatment & $1(9.1)$ & $0(0)$ \\
\hline Adverse drug reactions & $10(90.9)$ & $3(75)$ \\
\hline Serious adverse drug reactions & $1(9.1)$ & $2(50)$ \\
\hline
\end{tabular}




\begin{tabular}{|c|c|c|}
\hline & Tocilizumab & Placebo \\
\hline & Case (\%) & Case (\%) \\
\hline Adverse drug reactions leading to withdrawal from treatment & $1(9.1)$ & $0(0)$ \\
\hline
\end{tabular}

The median serum TCZ concentration increased from $23.80 \mu \mathrm{g} / \mathrm{mL}$ (range, $11.4-48.1 \mu \mathrm{g} / \mathrm{mL}$ ) at week 4 to $39.20 \mu \mathrm{g} / \mathrm{mL}$ (range, $21.4-83.9 \mu \mathrm{g} / \mathrm{mL}$ ) at week 24 , and the trough concentration remained around 20 $\mu \mathrm{g} / \mathrm{mL}$ after week 4 . No anti-TCZ antibodies were detected in any patient after the start of TCZ treatment.

\section{Discussion}

In this randomized, double-blind, placebo-controlled trial, although there was no statistically significant difference in the primary endpoint (the number of fever attacks), the TCZ group was more effective than the placebo group in most endpoints. During the double-blind period, 10 of 13 fever attacks in the TCZ group and 11 of 20 in the placebo group were identified by week 8 , and there was a trend toward suppression of fever attacks in the TCZ group compared to the placebo group as the treatment period increased. This result may indicate that the attacks appeared before the blood concentration of TCZ stabilized and the drug effect was fully exerted. In line with this observation, the percentage of patients who entered the rescue phase was higher in the placebo group compared to that of the TCZ group. In the placebo group, there were more patients who required intervention for increased fever attacks after week 14. The failure to validate the effect of TCZ in the primary endpoint was impacted by the lower-thanexpected estimated number of fever attacks in the placebo group ( 0.113 per week). This was influenced by the small number of patients, given that half of the patients in the placebo group were attack-free.

The results of the subsequent open-label extension study showed that the numbers of fever attacks and accompanying symptoms, such as pleurisy and peritonitis, tended to be generally controlled in the long term both in patients who continued to receive TCZ from the preceding study and in those who switched to TCZ. Consistent with the results of this study, there are many reports of efficacy in the literature, including case reports of TCZ [19-22, 30, 31].

Currently, IL-1 inhibitors, including canakinumab, have been shown to be effective in treating crFMF [32, 33], but in some cases, they are not effective enough or cannot be used due to adverse events. IL-1 inhibitors have also been shown to be effective against amyloidosis [34, 35]. It has been proposed that IL6 inhibitors may have a higher potential to normalize SAA than other biologic agents [36] and IL-6 inhibitors have been suggested to be useful in preventing the progression of amyloidosis and improving amyloid deposition [21, 37].

In the double-blind period, 2 serious adverse events were observed in 2 patients in the TCZ group (myocarditis, headache) and 1 in the placebo group (hypoglycemia). The following is the detailed history of a case of myocarditis. After 4 weeks of treatment with the investigational drug, the patient developed 
chest pain, mild ST-segment elevation at V1-4 on electrocardiogram, and cardiac enlargement on plain chest radiograph. Therefore, the patient was evaluated by cardiac catheterization. The catheterization showed no evidence of infarction and myocarditis was suspected, so a myocardial biopsy was performed, which showed neutrophilic inflammation. The cardiologist suggested systemic administration of steroids for progressive myocarditis. Since it was difficult to evaluate this study under moderate doses of steroids, the investigator decided to discontinue the study drug. The myocarditis improved with prednisolone $30 \mathrm{mg}$ oral administration, and prednisone was tapered off. Except for the case of myocarditis, there was no causal relationship with the investigational drug, and the patients recovered with treatment. Although the duration of treatment and the numbers of patients in the prior and continuing studies are limited, the safety profile of TCZ in our research is similar to that in the information available to date [38,39], and TCZ is considered to be well tolerated in patients with FMF.

There are several limitations to this study. First, the sample size was small, resulting in low statistical power and uncertainty in the results. Second, for patients who did not have fever attacks during the observation period, it was assumed that the frequency of fever attacks would remain low after entry, and therefore, reconsent for the same patients should not be allowed. Third, we should have considered an analysis in which attacks up to 4-8 weeks, when blood levels of TCZ were stable, were not included in the evaluation. Fourth, the high response to placebo could be attributed to differences in the baseline colchicine doses or the number of fever attacks during 24 weeks prior to study entry. Fifth, the percentage of Japanese patients with MEFV exon 10 variants is lower than those in Western countries [40-42], and the number of participants with exon 10 variants was small in this study. The overall low frequency of attacks in this study may be due to the small number of cases with the pathogenic variants in exon 10 , which reflects the genetic characteristics of FMF in Japan. Despite these limitations, this is the first double-blind, randomized, placebo-controlled trial to demonstrate the long-term efficacy of TCZ in reducing recurrent fever attacks, and the results of this study provide useful insight into the management of FMF.

\section{Conclusions}

In conclusion, administration of TCZ to FMF patients with inadequate or intolerant responses to colchicine was suggested to be effective in reducing the numbers of fever attacks and accompanying symptoms associated with FMF, and our results suggested that TCZ showed stable efficacy even after long-term administration. Additionally, no new concerns about safety have been found. Based on these results, TCZ may be a useful treatment option for FMF patients who have had an inadequate response to existing therapies.

\section{Abbreviations}

Cls

confidence intervals

FMF

Page $13 / 21$ 
Familial Mediterranean fever

IL

interleukin

IQRs

interquartile ranges

SDs

standard deviations

SF-36

the 36 -item short form health survey

TCZ

tocilizumab

TNF-a

necrosis factor-alpha

VAS

Visual Analog Scale

\section{Declarations}

\section{Acknowledgments}

The authors would like to thank our colleagues and staff at the Rheumatology Department of Nagasaki University Hospital and clinical research coordinators at all participating center for their support.

\section{Funding}

The study is funded by the Japan Agency for Medical Research and Development (Grant no. 17824542 to AK). Partial financial support was provided by Chugai Pharmaceutical Co., Ltd. for the NUH02FMF study.

\section{Availability of data and materials}

The datasets used and/or analyzed during the present study are available from the corresponding author on reasonable request.

\section{Author Contributors}

TK, SS, NH, HY and AK contributed to the conception and design of this study. TK, MI, TY, YK, KI, AY, KM, DK, TA and AK recruited patients into the study and participated in data collection. TK and SS contributed to the data analysis. All authors contributed to data interpretation, critically reviewed the article for important intellectual content and approved the final draft for submission.

\section{Ethics approval and consent to participate}


The study was approved by each center's institutional review board or ethics committee. All patients gave signed informed consent.

\section{Competing interests}

The investigational drugs (TCZ and placebo) and the TCZ drug information were provided by Chugai Pharmaceutical Co., Ltd. The authors declare that they have no competing interests.

\section{References}

1. Ben-Chetrit E, Levy M: Familial Mediterranean fever. Lancet 1998, 351(9103):659-664.

2. Federici S, Sormani MP, Ozen S, Lachmann HJ, Amaryan G, Woo P, Kone-Paut I, Dewarrat N, Cantarini $\mathrm{L}$, Insalaco A et al: Evidence-based provisional clinical classification criteria for autoinflammatory periodic fevers. Ann Rheum Dis 2015, 74(5):799-805.

3. Stehlik C, Reed JC: The PYRIN connection: novel players in innate immunity and inflammation. $J$ Exp Med 2004, 200(5):551-558.

4. Touitou I: The spectrum of Familial Mediterranean Fever (FMF) mutations. European journal of human genetics: EJHG 2001, 9(7):473-483.

5. Booty MG, Chae JJ, Masters SL, Remmers EF, Barham B, Le JM, Barron KS, Holland SM, Kastner DL, Aksentijevich I: Familial Mediterranean fever with a single MEFV mutation: where is the second hit? Arthritis Rheum 2009, 60(6):1851-1861.

6. Zemer D, Livneh A, Danon YL, Pras M, Sohar E: Long-term colchicine treatment in children with familial Mediterranean fever. Arthritis Rheum 1991, 34(8):973-977.

7. Wright DG, Wolff SM, Fauci AS, Alling DW: Efficacy of intermittent colchicine therapy in familial Mediterranean fever. Annals of internal medicine 1977, 86(2):162-165.

8. van der Hilst J, Moutschen M, Messiaen PE, Lauwerys BR, Vanderschueren S: Efficacy of anti-IL-1 treatment in familial Mediterranean fever: a systematic review of the literature. Biologics: targets \& therapy 2016, 10:75-80.

9. Hara K, Koga T, Endo Y, Sumiyoshi R, Furukawa K, Kawakami A: Genetic and clinical characteristics associated with efficacy and retention rates of colchicine in Japanese patients with familial Mediterranean fever: A single-center observational study. Mod Rheumatol 2020:1-2.

10. Oktem S, Yavuzsen TU, Sengul B, Akhunlar H, Akar S, Tunca M: Levels of interleukin-6 (IL-6) and its soluble receptor (sIL-6R) in familial Mediterranean fever (FMF) patients and their first degree relatives. Clin Exp Rheumatol 2004, 22(4 Suppl 34):S34-36.

11. Manukyan GP, Ghazaryan KA, Ktsoyan Zh A, Tatyan MV, Khachatryan ZA, Hakobyan GS, Mkrtchyan VA, Kelly D, Coutts A, Aminov RI: Cytokine profile of Armenian patients with Familial Mediterranean fever. Clinical biochemistry 2008, 41(10-11):920-922.

12. Ben-Zvi I, Livneh A: Chronic inflammation in FMF: markers, risk factors, outcomes and therapy. Nature reviews Rheumatology 2011, 7(2):105-112. 
13. Koga T, Kawashiri SY, Migita K, Sato S, Umeda M, Fukui S, Nishino A, Nonaka F, Iwamoto N, Ichinose $\mathrm{K}$ et al: Comparison of serum inflammatory cytokine concentrations in familial Mediterranean fever and rheumatoid arthritis patients. Scandinavian journal of rheumatology 2018, 47(4):331-333.

14. Ugurlu S, Ergezen B, Egeli BH, Selvi O, Ozdogan H: Safety and efficacy of anti-interleukin-1 treatment in 40 patients, followed in a single centre, with AA amyloidosis secondary to familial Mediterranean fever. Rheumatology (Oxford) 2020, 59(12):3892-3899.

15. Koga T, Migita K, Kawakami A: Biologic therapy in familial Mediterranean fever. Mod Rheumatol 2016, 26(5):637-641.

16. Kuemmerle-Deschner JB, Gautam R, George AT, Raza S, Lomax KG, Hur P: A systematic literature review of efficacy, effectiveness and safety of biologic therapies for treatment of familial Mediterranean fever. Rheumatology (Oxford) 2020, 59(10):2711-2724.

17. Honda N, Yokogawa N, Koga T, Endo Y, Matsubara S: Protracted Febrile Myalgia Syndrome Treated With Tocilizumab. Journal of clinical rheumatology: practical reports on rheumatic \& musculoskeletal diseases 2020.

18. Yilmaz S, Tekgoz E, Cinar M: Recurrence of proteinuria after cessation of tocilizumab in patients with AA amyloidosis secondary to FMF. Eur J Rheumatol 2018, 5(4):278-280.

19. Umeda M, Aramaki T, Fujikawa K, Iwamoto N, Ichinose K, Terada K, Takeo G, Yonemitsu N, Ueki Y, Migita $\mathrm{K}$ et al: Tocilizumab is effective in a familial Mediterranean fever patient complicated with histologically proven recurrent fasciitis and myositis. International journal of rheumatic diseases 2015.

20. Yilmaz S, Cinar M, Simsek I, Erdem H, Pay S: Tocilizumab in the treatment of patients with AA amyloidosis secondary to familial Mediterranean fever. Rheumatology (Oxford) 2015, 54(3):564565.

21. Hamanoue S, Suwabe T, Hoshino J, Sumida K, Mise K, Hayami N, Sawa N, Takaichi K, Fujii T, Ohashi $K$ et al: Successful treatment with humanized anti-interleukin- 6 receptor antibody (tocilizumab) in a case of AA amyloidosis complicated by familial Mediterranean fever. Mod Rheumatol 2015:1-4.

22. Fujikawa K, Migita K, Tsukada T, Umeda M, Nonaka F, Kawakami A, Eguchi K: Interleukin-6 targeting therapy in familial Mediterranean fever. Clin Exp Rheumatol 2013, 31(3 Suppl 77):150-151.

23. Ugurlu S, Hacioglu A, Adibnia Y, Hamuryudan V, Ozdogan $\mathrm{H}$ : Tocilizumab in the treatment of twelve cases with aa amyloidosis secondary to familial mediterranean fever. Orphanet J Rare Dis 2017, 12(1):105.

24. Koga T, Sato S, Miyamoto J, Hagimori N, Kawazoe Y, Arinaga K, Fukushima C, Yamamoto H, Kawakami A: Comparison of the efficacy and safety of tocilizumab for colchicine-resistant or colchicine-intolerant familial Mediterranean fever: study protocol for an investigator-initiated, multicenter, randomized, double-blind, placebo-controlled trial. Trials 2018, 19(1):715.

25. Koga T, Hagimori N, Sato S, Morimoto S, Hosogaya N, Fukushima C, Yamamoto H, Kawakami A: An open-label continuation trial of tocilizumab for familial Mediterranean fever with colchicine 
ineffective or intolerance: Study protocol for investigator-initiated, multicenter, open-label trial. Medicine 2020, 99(1):e18328.

26. Livneh A, Langevitz P, Zemer D, Zaks N, Kees S, Lidar T, Migdal A, Padeh S, Pras M: Criteria for the diagnosis of familial Mediterranean fever. Arthritis Rheum 1997, 40(10):1879-1885.

27. Berkun $Y$, Eisenstein EM: Diagnostic criteria of familial Mediterranean fever. Autoimmunity reviews 2014, 13(4-5):388-390.

28. Ozen S, Sag E, Ben-Chetrit E, Gattorno M, Gul A, Hashkes PJ, Kone-Paut I, Lachmann HJ, Tsitsami E, Twilt $\mathrm{M}$ et al: Defining colchicine resistance/intolerance in patients with familial Mediterranean fever: a modified-Delphi consensus approach. Rheumatology (Oxford) 2020.

29. Hashkes PJ, Huang B: The familial Mediterranean fever (FMF) 50 score: does it work in a controlled clinical trial? Re-analysis of the trial of rilonacept for patients with colchicine-resistant or intolerant FMF. The Israel Medical Association journal: IMAJ 2015, 17(3):137-140.

30. Colak S, Tekgoz E, Cinar M, Yilmaz S: The assessment of tocilizumab therapy on recurrent attacks of patients with familial Mediterranean fever: A retrospective study of 15 patients. Mod Rheumatol 2021, 31(1):223-225.

31. Aikawa E, Shimizu T, Koga T, Endo Y, Umeda M, Hori T, Irie J, Kuroda K, Eguchi M, Okamoto M et al: Atypical Familial Mediterranean Fever Complicated with Gastrointestinal Amyloidosis Diagnosed due to Paroxysmal Arthralgia and Intractable Diarrhea, Successfully Treated with Tocilizumab. Intern Med 2019, 58(12):1781-1785.

32. Sag E, Akal F, Atalay E, Akca UK, Demir S, Demirel D, Batu ED, Bilginer Y, Ozen S: Anti-IL1 treatment in colchicine-resistant paediatric FMF patients: real life data from the HELIOS registry. Rheumatology (Oxford) 2020, 59(11):3324-3329.

33. Ozen S, Ben-Cherit E, Foeldvari I, Amarilyo G, Ozdogan H, Vanderschueren S, Marzan K, Kahlenberg $J M$, Dekker $E$, De Benedetti $F$ et al: Long-term efficacy and safety of canakinumab in patients with colchicine-resistant familial Mediterranean fever: results from the randomised phase III CLUSTER trial. Ann Rheum Dis 2020, 79(10):1362-1369.

34. Sozeri $B$, Gulez N, Ergin M, Serdaroglu E: The experience of canakinumab in renal amyloidosis secondary to Familial Mediterranean fever. Mol Cell Pediatr 2016, 3(1):33.

35. Varan O, Kucuk H, Babaoglu H, Guven SC, Ozturk MA, Haznedaroglu S, Goker B, Tufan A: Efficacy and safety of interleukin-1 inhibitors in familial Mediterranean fever patients complicated with amyloidosis. Mod Rheumatol 2019, 29(2):363-366.

36. Okuda Y: AA amyloidosis - Benefits and prospects of IL-6 inhibitors. Mod Rheumato/2019, 29(2):268-274.

37. Lane T, Gillmore JD, Wechalekar AD, Hawkins PN, Lachmann HJ: Therapeutic blockade of interleukin6 by tocilizumab in the management of $A A$ amyloidosis and chronic inflammatory disorders: a case series and review of the literature. Clin Exp Rheumatol 2015, 33(6 Suppl 94):S46-53.

38. Burmester GR, Rubbert-Roth A, Cantagrel A, Hall S, Leszczynski P, Feldman D, Rangaraj MJ, Roane G, Ludivico C, Bao M et al: Efficacy and safety of subcutaneous tocilizumab versus intravenous 
tocilizumab in combination with traditional DMARDs in patients with RA at week 97 (SUMMACTA). Ann Rheum Dis 2016, 75(1):68-74.

39. Ogata A, Tanimura $K$, Sugimoto $T$, Inoue $H$, Urata $Y$, Matsubara $T$, Kondo $M$, Ueki $Y$, Iwahashi $M$, Tohma S et al: Phase III study of the efficacy and safety of subcutaneous versus intravenous tocilizumab monotherapy in patients with rheumatoid arthritis. Arthritis Care Res (Hoboken) 2014, 66(3):344-354.

40. Migita $\mathrm{K}$, Ida $\mathrm{H}$, Moriuchi $\mathrm{H}$, Agematsu K: Clinical relevance of MEFV gene mutations in Japanese patients with unexplained fever. J Rheumatol 2012, 39(4):875-877.

41. Kirino Y, Zhou Q, Ishigatsubo Y, Mizuki N, Tugal-Tutkun I, Seyahi E, Ozyazgan Y, Ugurlu S, Erer B, Abaci $\mathrm{N}$ et al: Targeted resequencing implicates the familial Mediterranean fever gene MEFV and the toll-like receptor 4 gene TLR4 in Behcet disease. Proc Natl Acad Sci U S A 2013, 110(20):8134-8139.

42. Endo Y, Koga T, Ishida M, Fujita Y, Tsuji S, Takatani A, Shimizu T, Sumiyoshi R, Igawa T, Umeda M et al: Musculoskeletal manifestations occur predominantly in patients with later-onset familial Mediterranean fever: Data from a multicenter, prospective national cohort study in Japan. Arthritis Res Ther 2018, 20(1):257.

\section{Figures}




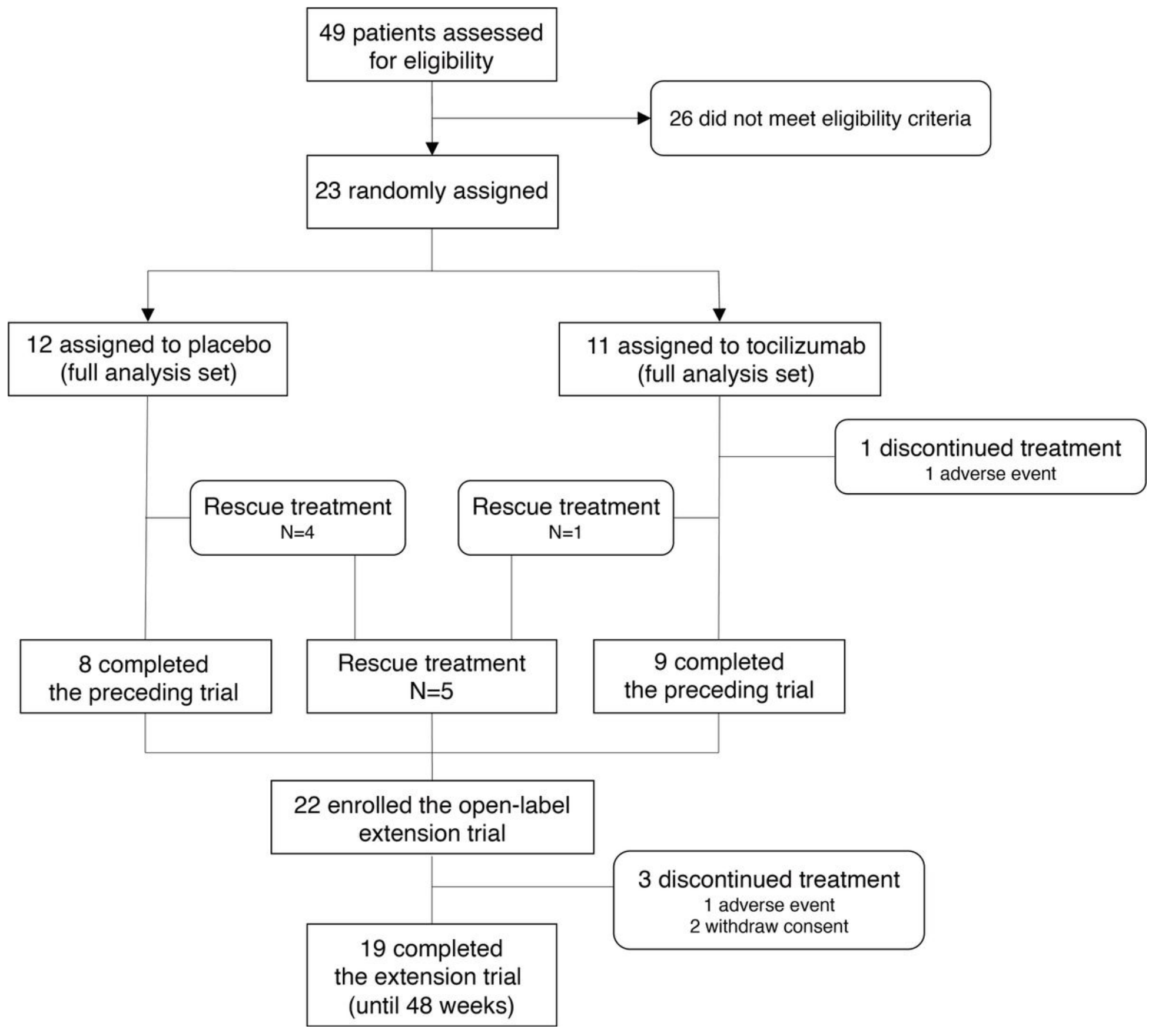

Figure 1

Trial profile 
Tocilizumab

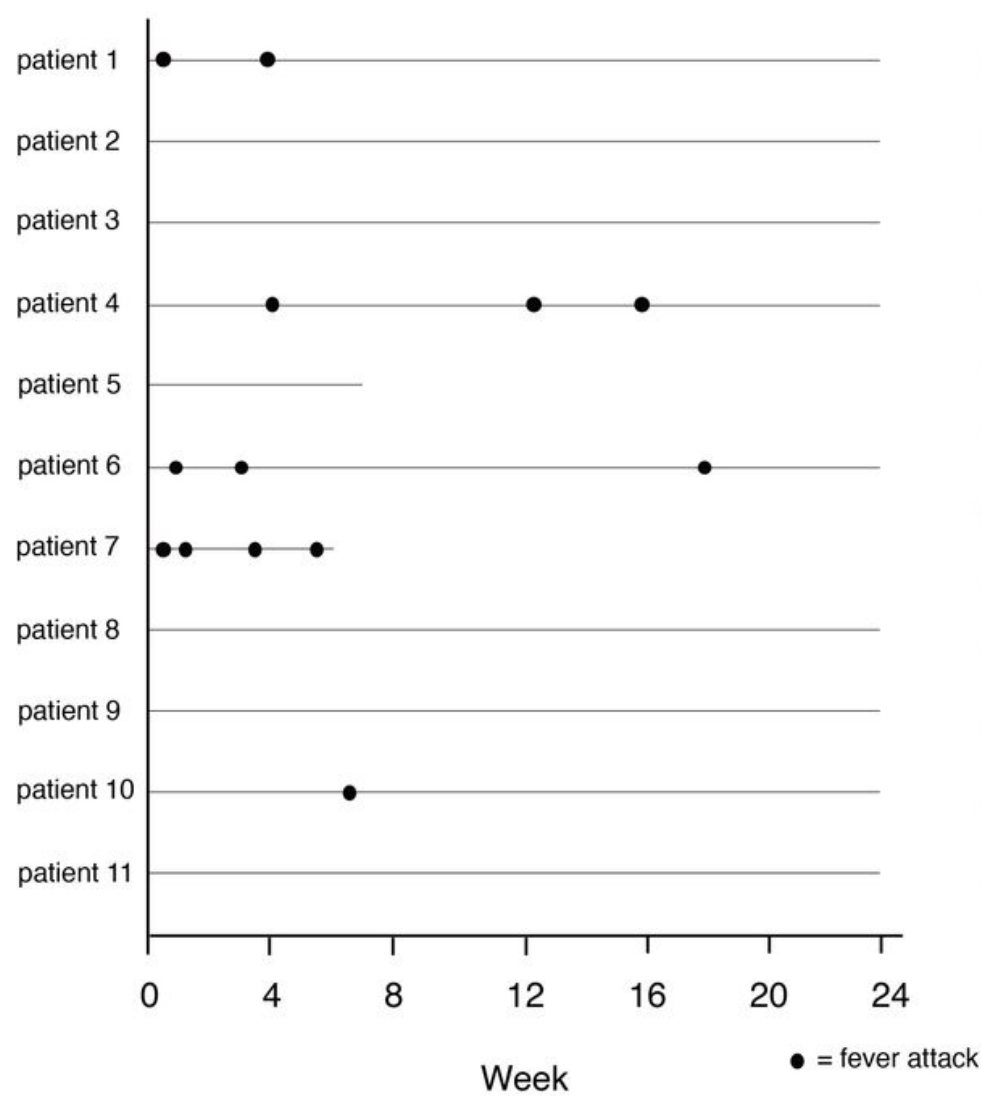

Placebo

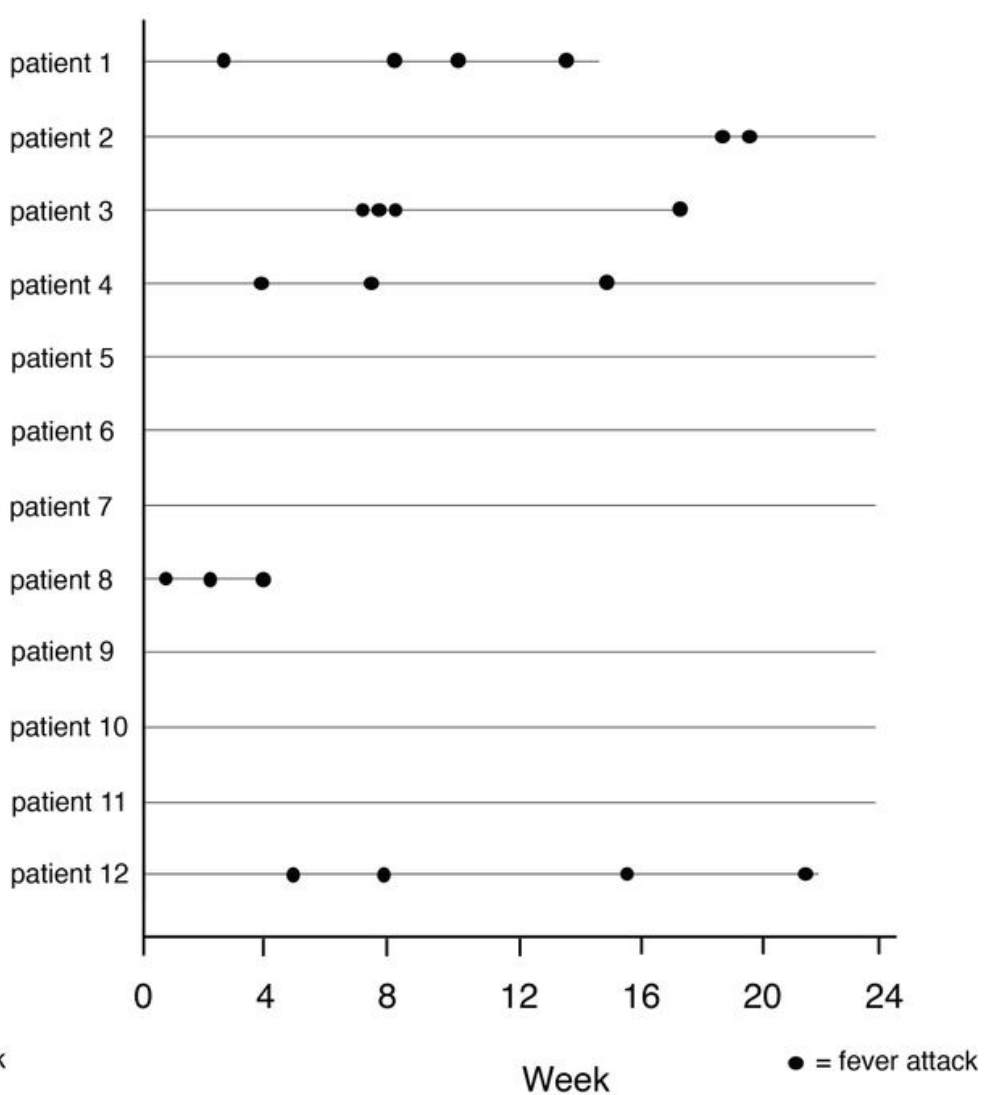

Figure 2

Dot plot of fever attacks per patient in the double-blind period in the full analysis set (NUH01FMF)
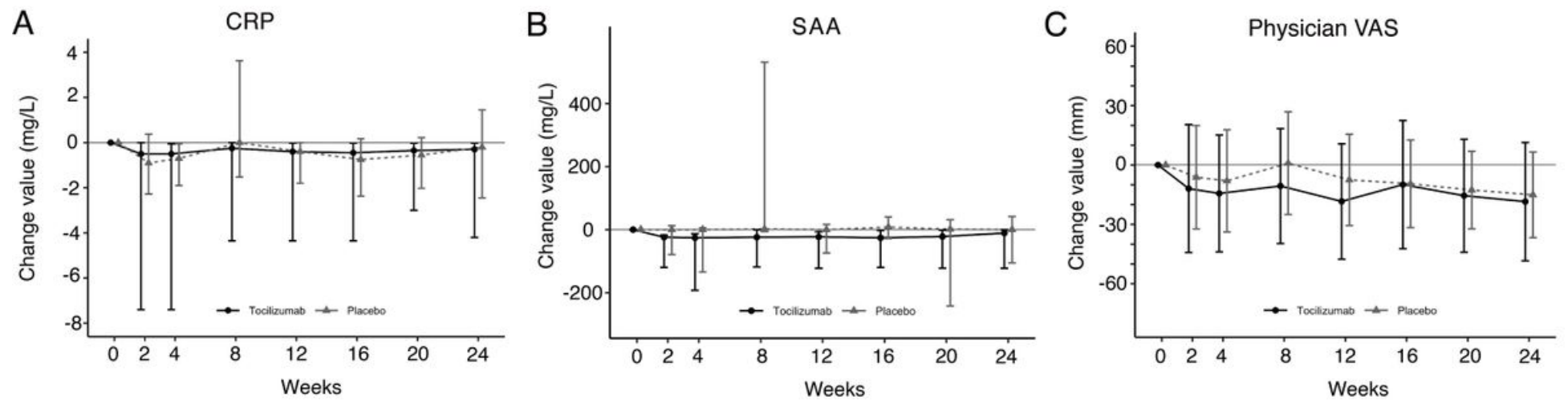

Figure 3

Results from the full analysis set (NUH01FMF) for (A) changes in serum $\mathrm{C}$ reactive protein (CRP) from baseline, $(B)$ changes in serum amyloid $A(S A A)$ from baseline, $(C)$ changes in physician VAS from baseline. Points are median (A and $B$ ) or mean $(C)$. Bars are IQR (A and B) or SD (C).

\section{Supplementary Files}

This is a list of supplementary files associated with this preprint. Click to download. 
- Supplementarytable1.docx

- supplementaryTable2.docx

- Supplementarytable3.docx

- 210618sFigureS1.jpg

- 210618sup.FigureS2.jpg 